\title{
INSCRIBED AND CIRCUMSCRIBED POLYHEDRA FOR A CONVEX BODY AND CONTINUOUS FUNCTIONS ON A SPHERE IN EUCLIDEAN SPACE
}

\author{
V. V. MAKEEV
}

\begin{abstract}
Two related problems concerning continuous functions on a sphere $S^{n-1}$ $\subset \mathbb{R}^{n}$ are studied, together with the problem of finding a family of polyhedra in $\mathbb{R}^{n}$ one of which is inscribed in (respectively, circumscribed about) a given smooth convex body in $\mathbb{R}^{n}$. In particular, it is proved that, in every convex body $K \subset \mathbb{R}^{3}$, one can inscribe an eight-vertex polyhedron obtained by "equiaugmentation" of a similarity image of any given tetrahedron of class $T$.
\end{abstract}

\section{$\S 1$. INTRODUCTION}

In the present paper, we study two related problems concerning continuous functions on a sphere $S^{n-1} \subset \mathbb{R}^{n}$ and also the problem of finding a family of polyhedra in $\mathbb{R}^{n}$ one of which is inscribed in (respectively, circumscribed about) a given smooth convex body in $\mathbb{R}^{n}$. In the sequel, by a convex body, we mean a compact convex subset $K$ of $\mathbb{R}^{n}$ with nonempty interior. We describe the above-mentioned problems in more detail.

The problem on continuous functions on the standard sphere $S^{n-1} \subset \mathbb{R}^{n}$ consists in finding families $M$ of sets of points on the sphere such that one of the sets can be placed in a level set of any given continuous function on the sphere (or of several functions simultaneously).

In the best known case of this problem, the set $M$ consists of the images of a set of points $\left\{A_{1}, \ldots, A_{k}\right\}$ in $S^{n-1} \subset \mathbb{R}^{n}$ under all possible rotations of the sphere. In this case, we must find all configurations of points $A_{1}, \ldots, A_{k} \in S^{n-1} \subset \mathbb{R}^{n}$ such that, for every continuous mapping $f: S^{n-1} \rightarrow \mathbb{R}^{m}$, there is a rotation $a$ of the sphere with $f\left(a\left(A_{1}\right)\right)=\cdots=f\left(a\left(A_{k}\right)\right)$.

Knaster [1] conjectured that every set of points on the sphere has this property if $k+m=n+1$.

It has long been known that the Knaster conjecture is true for $k=2$ (and for an arbitrary $n$ and $m=n-1$ ). Floyd [2] proved this conjecture for $n=3$.

As we know now [3]-[6], the conjecture is not valid in most of the remaining cases except, possibly, for some small values of $n$ and in the case where $k=3$.

Another well-known problem is to find a family of polyhedra universally inscribed in or circumscribed about smooth convex bodies in $\mathbb{R}^{n}$. In other words, to find a family $\tilde{M}$ of polyhedra in $\mathbb{R}^{n}$ such that, for every smooth convex body $K \subset \mathbb{R}^{n}$, there is a polyhedron $m \in \tilde{M}$ inscribed in $K$ (circumscribed about $K$ ).

In the best known examples, the set $\tilde{M}$ consists of the images of a given polyhedron in $\mathbb{R}^{n}$ under homothety, similarity, or affine transformations. For a survey of results, see $[7]-[9]$. 
The two above-mentioned problems are connected; in some cases, knowing solutions of one problem, we can construct solutions of the other one.

Thus, in [10], we use solutions of the problem on continuous functions on the twodimensional sphere for constructing families of quadrangles that can be inscribed into every smooth star Jordan curve in a plane. However, few nontrivial solutions of the "Knaster problem" on continuous functions on a sphere are known, which makes it difficult to use this method.

Below, we also consider a more general problem, in which, for given points on $S^{n-1} \subset$ $\mathbb{R}^{n}$ and a given continuous function on the sphere, we obtain, by rotating the sphere, linear relations with coefficients \pm 1 for the values of the function at these points.

We say that a field of convex bodies (resp., figures) (f.c.b., resp., f.c.f.) is defined in a vector bundle $\gamma: E \rightarrow B$ if, in every fiber, we have a convex body (figure) that continuously depends on the fiber. In the sequel, by $\gamma_{k}^{n}: E_{k}\left(\mathbb{R}^{n}\right) \rightarrow G_{k}\left(\mathbb{R}^{n}\right)$, we denote the tautological bundle on the Grassmann manifold $G_{k}\left(\mathbb{R}^{n}\right)$ of $k$-planes passing through $0 \in \mathbb{R}^{n}$, in which the fiber over a plane is the same plane regarded as a $k$-dimensional subspace of $\mathbb{R}^{n}$. The spherization of the bundle $\gamma_{3}^{n}$, i.e., its restriction to the set of unit vectors in $E_{3}\left(\mathbb{R}^{n}\right)$, is denoted by $S_{3}^{n}: S_{3}\left(\mathbb{R}^{n}\right) \rightarrow G_{3}\left(\mathbb{R}^{n}\right)$. As usual, by typical points in a topological space, we mean the points of an open dense subset in this space.

\section{§2. INSCRIBED EIGHT-VERTEX POLYHEDRA AND CIRCUMSCRIBED OCTAHEDRONS IN $\mathbb{R}^{3}$ AND THE PROBLEM ON CONTINUOUS FUNCTIONS ON A THREE-DIMENSIONAL UNIT SPHERE}

By a twisted prism, we mean a convex hull of two equal squares lying in planes perpendicular to the line passing through their centers. The midpoint of the segment connecting the centers of the bases of a twisted prism is called the center of the prism.

In [17], it is proved that, in every sphere $S^{2}$ smoothly embedded in $\mathbb{R}^{3}$, and, therefore, in every smooth convex body $K \subset \mathbb{R}^{3}$, one can inscribe a twisted prism with a prescribed ratio of a base edge to the height.

The dual fact is also valid: about every bounded subset of $\mathbb{R}^{3}$, one can circumscribe an octahedron obtained by twisting a regular quadrangular bipyramid with a prescribed ratio of the height to the base edge. By twisting, we mean a simultaneous rotation of all four faces bounding the pyramid and passing through its vertex about the height of the bipyramid.

It turns out that, for a typical smooth body in $\mathbb{R}^{3}$, there exists an odd number of required inscribed twisted prisms and an odd number of circumscribed twisted bipyramids. In both cases, as a typical body $K \subset \mathbb{R}^{3}$, we can take a heteraxial ellipsoid (for inscribed twisted prisms, see [17]).

Theorem 1. Reassume that, for $n \geq 4$, we have $n-3$ f.c.b. in the bundle $\gamma_{3}^{n}: E_{3}\left(\mathbb{R}^{n}\right) \rightarrow$ $G_{3}\left(\mathbb{R}^{n}\right)$ such that, in each field, every body has zero as an interior point in its fiber. Let $x_{1}, \ldots, x_{n-3}$ be arbitrary positive real numbers. Then there is a fiber $E \subset E_{3}\left(\mathbb{R}^{n}\right)$ such that, for $1 \leq i \leq n-3$, in the corresponding body of the ith field, one can inscribe a twisted prism with center at zero for which the ratio of the base edge to the height is equal to $x_{i}$.

Proof. We prove the theorem for smooth fields of convex bodies.

For $1 \leq i \leq n-3$, we denote by $\tilde{\gamma}_{i}^{n}: \widetilde{E_{i}\left(\mathbb{R}^{n}\right)} \rightarrow G_{3}\left(\mathbb{R}^{n}\right)$ the bundle associated with $\gamma_{3}^{n}$ in which the fiber over a 3-plane from $G_{3}\left(\mathbb{R}^{n}\right)$ is the manifold of twisted prisms lying in this plane and having the ratio of the base edge to the height equal to $x_{i}$. Let $\pi_{i}: \widetilde{E_{i}\left(\mathbb{R}^{n}\right)} \rightarrow E_{3}\left(\mathbb{R}^{n}\right)$ be the projection sending a twisted prism to its center. 


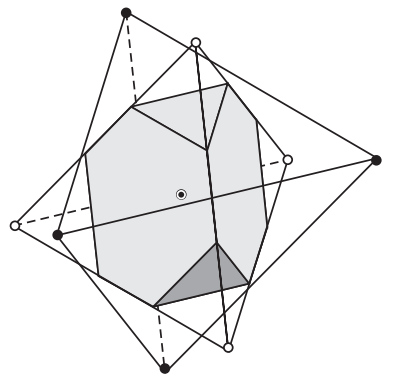

FIGURE 1

For $1 \leq i \leq n-1$, we denote by $C_{i}$ the set of twisted prisms in $\widetilde{E_{i}\left(\mathbb{R}^{n}\right)}$ inscribed in the bodies of the $i$ th of the f.c.b. in $\gamma_{3}^{n}$ under consideration. For an f.c.b. in general position, the $C_{i}$ are smooth compact submanifolds in $\widetilde{E_{i}\left(\mathbb{R}^{n}\right)}$, and, as follows from the above, they intersect the typical fibers in an odd number of points.

Then the $\pi_{i}\left(C_{i}\right)$ realize the cycles modulo 2 that intersect the typical fibers of $E_{3}\left(\mathbb{R}^{n}\right)$ in an odd number of points. Let $C_{0}$ be the cycle realized by the zero section of the bundle $\gamma_{3}^{n}$. It is well known that the intersection number for the cycles $C_{0}, \pi C_{1}, \ldots, \pi C_{n-3}$ is 1 modulo 2 [13]-[16], which completes the proof of Theorem 1 in the case of the general position. In the other cases, the theorem is proved by passing to the limit.

Theorem 2. Let $n \geq 4$, let $f_{1}, \ldots, f_{n-3}$ be continuous functions given on the spherization $S_{3}^{n}: S_{3}\left(\mathbb{R}^{n}\right) \rightarrow G_{3}\left(\mathbb{R}^{n}\right)$ of the tautological bundle, and let $x_{1}, \ldots, x_{n-3}$ be $n-3$ positive numbers. Then there exists a fiber $S^{2} \hookrightarrow S_{3}\left(\mathbb{R}^{n}\right)$ of the bundle in which $n-3$ twisted prisms are inscribed so that, for $1 \leq i \leq n-3$, the function $f_{i}$ takes equal values at the vertices of the ith prism and the ratio of the base edge to the height of the ith prism is equal to $x_{i}$.

Proof. As in the proof of Theorem 1, we prove that, in general position, there is an odd number of the required configurations of prisms.

To this end, we consider $n-3$ smooth f.c.b. in $\gamma_{3}^{n}$ as in the statement of Theorem 1 . We define functions $f_{i}$ on $S_{3}\left(\mathbb{R}^{n}\right)$ by putting $f_{i}(x)$ equal to the length of the segment cut out by the body of the $i$ th field from the ray $O x$ in the corresponding fiber. Now, from the proof of Theorem 1, we obtain that, in the general position, the number of the required twisted prisms is odd. In the remaining cases, the theorem is proved by passing to the limit.

From Theorem 2, we obtain the following statement.

Corollary 1. For every positive number $x$ and every continuous function $f$ on the Standard sphere $S^{3} \hookrightarrow \mathbb{R}^{4}$, there exists a hyperplane central cross section of $S^{3}$ in which one can inscribe a twisted prism at the vertices of which the function $f$ takes equal values and the ratio of the base edge of which to the height is equal to $x$.

In the sequel, by $T$ we denote the class of tetrahedra $A B C D$ in $\mathbb{R}^{3}$ such that $|A B|=$ $|C D|$ and $|A C|=|C B|=|B D|=|D A|$ (i.e., the tetrahedra with equal isosceles faces).

By an equitruncated tetrahedron, we mean the intersection of two negatively homothetic tetrahedra with common center of mass (see Figure 1).

By equiaugmentation of a tetrahedron, we mean the convex hull of two negatively homothetic tetrahedra with common center of mass (see Figure 2). As V. I. Subbotin pointed out, a regular equiaugmented tetrahedron is sometimes called a trigonal tritetrahedron. 


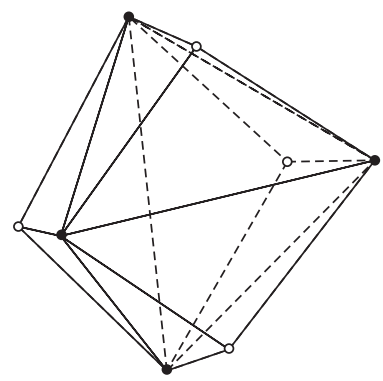

FiguRE 2

Theorem 3. About every bounded subset $K \subset \mathbb{R}^{3}$ one can circumscribe an octahedron obtained by "equitruncating" a similarity image of a given tetrahedron of class $T$.

Proof. All faces of the above tetrahedron are equal isosceles triangles. Therefore, the lengths of the heights of the tetrahedron are equal. The heights of the tetrahedron in question are parallel to the diagonals of a certain regular quadrangular prism.

Therefore, the statement of Theorem 1 is equivalent to the fact that the body $K$ has the same width in the direction of the four diagonals of some regular quadrangular prism. This follows, for example, from the fact [18, Theorem 1] that, for the vertices $A, B, C$, and $D$ of a square on the standard sphere $S^{2} \hookrightarrow \mathbb{R}^{3}$ and an even continuous function $f$ on the sphere (i.e., $f(-x)=f(x)$ for $x \in S^{2}$ ), there is a rotation $a$ of the sphere such that $f(a(A))=f(a(B))=f(a(C))=f(a(D))$.

Remarks. 1. If $K$ is centrally symmetric, then the equitruncated circumscribed tetrahedron constructed above is automatically centrally symmetric, and, therefore, is a regular biprism with a given ratio of edges.

2. It seems that nothing contradicts the assumption that the class of tetrahedra in Theorem 1 can be extended to tetrahedra with equal faces (the case where $K$ is a ball shows that this condition is necessary). However, the author does not know how to prove this.

3. By analogy with tetrahedra, we can consider equitruncated and equiaugmented simplices in $\mathbb{R}^{n}$. It seems very likely that about every bounded subset $K$ in $\mathbb{R}^{n}$ one can circumscribe a regular equitruncated simplex (or even a simplex with faces of the same $(n-1)$-dimensional measure).

Theorem 4. For every convex body $K \subset \mathbb{R}^{3}$, one can inscribe an eight-vertex polyhedron obtained by equiaugmentation of a similarity image of an arbitrary tetrahedron of class $T$.

Proof. We prove that, for a typical smooth convex body $K \subset \mathbb{R}^{3}$ (i.e., for an open set of smooth convex bodies $K \subset \mathbb{R}^{3}$ that is dense in the $C^{1}$-topology), the number of required polyhedra is odd.

Up to similarity, the required eight-vertex polyhedra form a one-parameter family and the group of similarities of $\mathbb{R}^{3}$ is seven-dimensional. Since we have eight conditions for a vertex to lie on the boundary of $K \subset \mathbb{R}^{3}$, we see that, for a typical smooth body $K$, there is a finite number of required inscribed eight-vertex polyhedra.

By standard topological considerations and the fact that an eight-vertex polyhedron inscribed in a convex body cannot degenerate under a smooth deformation of the body, we see that, for a typical smooth convex body $K \subset \mathbb{R}^{3}$, the residue modulo 2 of the number of required inscribed eight-vertex polyhedra does not depend on the body. We prove that this residue is 1 . 
As a convex body $K \subset \mathbb{R}^{3}$, we take (as in $[17,18,8]$ ) a heteraxial ellipsoid. Since the locus of the endpoints of the chords of $K$ passing through a fixed interior point distinct from the center of $K$ that divides the chords in a fixed ratio is planar, we see that each required eight-vertex polyhedron inscribed in $K$ automatically has a center (a common center of the two tetrahedra with the convex hull of which it coincides) at the center of the ellipsoid $K$. Therefore, in the case in question, the required eight-vertex polyhedra are regular quadrangular prisms with center at the center of $K$ and a given ratio of edges. In [17] and [18], it is proved that there are three such prisms if the prisms are not cubes and one prism if the prisms are cubes.

The fact that the heteraxial ellipsoid $K$ is typical in the sense defined more precisely below can easily be visualized. Let $\ell_{1}, \ell_{2}, \ell_{3}$, and $\ell_{4}$ be the lines passing through the center of an eight-vertex polyhedron and the pairs of its opposite vertices. From the above, it follows that if we move the common point of the lines $\ell_{1}, \ldots, \ell_{4}$ with nonzero speed from the center of the ellipsoid $K$, then, for the lines $\ell_{i}$, the condition that the ratios of the segments cut out of the lines by the ellipsoid $K$ (counted from the common origin of the lines) is violated by a nonzero speed. If we rotate the set of lines about their common point (the center of the ellipsoid $K$ ), then one of the equality conditions for the segments cut out of $\ell_{i}$ by $K$ is violated by nonzero speed, as shown, e.g., in [17].

Thus, Theorem 4 is proved for an open subset of smooth convex bodies $K \subset \mathbb{R}^{3}$ dense in the $C^{1}$-topology. For an arbitrary convex body $K$, we prove Theorem 2 by passing to the limit, which needs justification.

Let $K_{n}$ be a sequence of typical smooth convex bodies convergent to a convex body $K \subset \mathbb{R}^{3}$ in the Hausdorff metric. We proved that an equiaugmented tetrahedron $T_{n}$ can be inscribed in $K_{n}$. By the standard compactness considerations, there is a subsequence of $T_{n}$ that converges either to an equiaugmented tetrahedron inscribed in $K$ or to a point. It remains to prove that $T_{n}$ does not have a subsequence of eight-vertex polyhedra that converges to a point.

Below, for simplicity, we consider the case of a regular equiaugmented tetrahedron.

Lemma 1. Let $k$ be the similarity coefficient for tetrahedra forming a regular equiaugmented tetrahedron $T$. If $|k-1| \geq \varepsilon>0$, then there is a constant $C(\varepsilon)$ such that the ratio of the diameter of the convex body $K$ circumscribed about $T$ to the diameter of $T$ does not exceed $C(\varepsilon)$.

Proof. We assume the contrary. Then there exists a sequence of points $A_{n}$ going to infinity such that the convex hull $\left(T \cup\left\{A_{n}\right\}\right)$ is circumscribed about $T$. From the sequence of rays $0 A_{n}$, where 0 is the center of $T$, we choose a subsequence convergent to some ray. Then the union of the rays codirected with $\ell$ and originating from points of $T$ forms an unbounded body $K$ circumscribed about $T$. The body $\widetilde{K}$ polar to $K$ with respect to a ball centered at 0 (the center of $T$ ) is inscribed in the regular equitruncated tetrahedron $\widetilde{T}$ polar to $T$. Since the body $\widetilde{K}$ is bounded by the plane that passes through the point 0 and is perpendicular to the ray $\ell$, we see that $\widetilde{K}$ does not meet one of the triangular faces of $\widetilde{T}$. The contradiction obtained proves the lemma.

Thus, if a subsequence of $T_{n}$ converges to a point, then the similarity type of the eight-vertex polyhedra $T_{n}$ converges to the type of a cube.

If $T$ is a cube, then the width of the convex body $K \subset \mathbb{R}^{3}$ circumscribed about $T$ in the direction of one of its edges does not exceed three times the length of the edge of the cube.

Assuming the contrary, we see that the body $\widetilde{K}$ polar to $K$ is bounded by three planes that are perpendicular to edges of $T$, are at the distance less than $1 / 3$ of the length of 


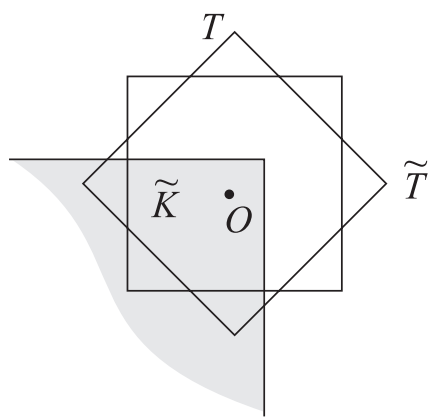

Figure 3

the edge of $T$ from its center, and do not meet one of the faces of the octahedron $\widetilde{T}$ polar to $T$ (see Figure 3 ).

For an eight-vertex polyhedron close to $T$, the number 3 in the above estimate can be replaced by a greater number. From the statements proved above, we see that a subsequence of eight-vertex polyhedra $T_{n}$ cannot converge to a point. Theorem 2 is proved.

Remarks. 1. Usually, it is more complicated to prove the possibility of inscribing a polyhedron of a certain type in a convex body than of circumscribing polyhedra about a bounded subset of a Euclidean space. We have seen that the proof of Theorem 2 is more complicated than that of Theorem 1. Moreover, usually, it is possible to inscribe polyhedra only in smooth convex bodies. For example, in [3], we proved that, in a smooth convex body $K \subset \mathbb{R}^{3}$, one can inscribe a similarity image of a given pentagonal pyramid, and the smoothness condition cannot be lifted. In [3], we also proved that, in an arbitrary smooth convex body, one can inscribe a regular octahedron. We note that the proof of the dual theorem of Kakutani [19] on the possibility of circumscribing a cube about every bounded subset of $\mathbb{R}^{3}$ is considerably easier. Thus, Theorem 2 is one of the few cases in which, when inscribing a polyhedron in a bounded body, we do not assume the smoothness of the body.

2. It seems very likely that, in every smooth convex body $K \subset \mathbb{R}^{n}$ for $n \geq 4$, one can inscribe a regular equiaugmented simplex. The author does not know how to prove this fact. For $n=2$, a similar fact is not valid.

Theorem 5. For every tetrahedron $t \in T$, every sphere $S^{2}$ smoothly embedded in $\mathbb{R}^{3}$ contains the vertices of some homothetic image of $t$ and also the vertices of the corresponding negatively homothetic image of $t$ with the same center of mass.

Proof. We prove that, for a typical smooth embedding $S^{2} \hookrightarrow \mathbb{R}^{3}$, the number of the required eight-vertex polyhedra is odd.

Under the condition that the centers of mass of the tetrahedra in question coincide, the manifold $M$ of the pairs $(A t,-\lambda A t)$, where $\lambda>0$ and $A$ is a similarity transformation is eight-dimensional. The eight conditions for the vertices of $A t$ and $-\lambda A t$ to lie on a typical embedded sphere $S^{2} \hookrightarrow \mathbb{R}^{3}$ give a finite number of the required pairs of tetrahedra the residue modulo 2 of which does not depend on the choice of a typical embedding.

Every two smooth embeddings $S_{0}^{2} \hookrightarrow \mathbb{R}^{3}$ and $S_{1}^{2} \hookrightarrow \mathbb{R}^{3}$ of a two-dimensional sphere are smoothly isotopic. Let $S_{t}^{2} \hookrightarrow \mathbb{R}^{3}$, where $0 \leq t \leq 1$, be the corresponding isotopy. The set $N$ of pairs $(A t,-\lambda A t)$ of tetrahedra inscribed in the spheres $S_{t}^{2} \hookrightarrow \mathbb{R}^{3}$ is compact since these tetrahedra are uniformly bounded. The family of spheres $S_{t}^{2} \hookrightarrow \mathbb{R}^{3}$ is smooth, and, therefore, the tetrahedra cannot degenerate. For a typical isotopy, the manifold $N$ 
is one-dimensional, compact, and smooth. The boundary of this manifold consists of the pairs $(A t,-\lambda A t)$ inscribed in the spheres $S_{0}^{2}$ and $S_{1}^{2}$, which implies the above-mentioned independence modulo 2.

This residue is calculated above, where we proved that it is equal to 1 for a heteraxial ellipsoid, which gives an example of a typical embedding $S^{2} \hookrightarrow \mathbb{R}^{3}$.

For an arbitrary smooth embedding $S^{2} \hookrightarrow \mathbb{R}^{3}$, the theorem is obtained by passing to the limit if we take into account the fact that, by smoothness, the limiting pair of inscribed polyhedra cannot degenerate.

By analogy with Theorem 1, we can prove the following statement.

Theorem 6. Reassume that, for $n \geq 4$, we have $n-3$ f.c.b. in the bundle $\gamma_{3}^{n}: E_{3}\left(\mathbb{R}^{n}\right) \rightarrow$ $G_{3}\left(\mathbb{R}^{n}\right)$ such that, in each field, every body has 0 as an interior point in its fiber. Let $t_{1}$, $\ldots, t_{n-3}$ be tetrahedra of class $T$. Then there exists a fiber $E \subset E_{3}\left(\mathbb{R}^{n}\right)$ such that, for $1 \leq i \leq n-3$, the boundary of the body of the ith field in this fiber contains the vertices of a similarity image of $t_{i}$ and also of the corresponding negatively homothetic tetrahedron with common center of mass at the point $0 \in \mathbb{R}^{n}$.

As above, Theorem 6 implies the following statement.

Theorem 7. Let $n \geq 4$, let $f_{1}, \ldots, f_{n-3}$ be continuous functions given on the spherization $S_{3}^{n}: S_{3}\left(\mathbb{R}^{n}\right) \rightarrow G_{3}\left(\mathbb{R}^{n}\right)$ of the tautological bundle, and let $t_{1}, \ldots, t_{n-3}$ be $n-3$ tetrahedra of class $T$. Then there exists a fiber $S^{2} \hookrightarrow S_{3}\left(\mathbb{R}^{n}\right)$ of the bundle in which, for $1 \leq i \leq n-3$, a similarity image of the tetrahedron $t_{i}$ is inscribed so that the function $f_{i}$ takes equal values at its vertices and also takes equal values at the points symmetric to its vertices with respect to the center of the fiber (the sphere $S^{2}$ ).

For $n=4$, we obtain the following statement.

Corollary 2. For every tetrahedron $t \in T$ and every continuous function $f$ on the sphere $S^{3} \hookrightarrow \mathbb{R}^{4}$, there is a central cross section of $S^{3}$ by a hyperplane $P$ such that, in $S^{3} \cap P$, one can inscribe a similarity image of $t$ at the vertices of which and also at the points symmetric to these vertices with respect to the center of $S^{3}$ the function $f$ takes equal values.

Proposition. Let $n \geq 4$, and let $f_{i}: S^{n-1} \hookrightarrow \mathbb{R}^{n}$ be $n-3$ smooth embeddings of the sphere. Let, for $1 \leq i \leq n-3$, the point $0 \in \mathbb{R}^{n}$ belong to the bounded component of $\mathbb{R}^{n} \backslash f_{i}\left(S^{n-1}\right)$. Then, for arbitrary $n-3$ given tetrahedra $t_{1}, \ldots, t_{n-3}$ of class $T$, there exist similarity images of $t_{1}, \ldots, t_{n-3}$ that have a common center at $0 \in \mathbb{R}^{n}$, lie in the same 3-plane, and are such that, for each $1 \leq i \leq n-3$, the images of the vertices of $t_{i}$ and of the negatively homothetic to it tetrahedron with the same center 0 lie on $f_{i}\left(S^{n-1}\right)$.

Proof. It is sufficient to observe that, as above, the number of the required configurations of tetrahedra is odd in the case of general position.

Since the smooth embeddings of spheres in question are smoothly isotopic, we can restrict ourselves to the typical case, in which $f_{i}\left(S^{n-1}\right)$ is a convex surface for $1 \leq i \leq$ $n-3$. In this case, the fact that the number of required configurations is odd is verified in the same way as in the proof of Theorem 1.

The following statement uses the above proposition and is its far-reaching generalization.

Theorem 8. Let $n \geq 4$, and let $K_{1}, \ldots, K_{n-3}$ be compact subsets of $\mathbb{R}^{n}$ each of which subdivides $\mathbb{R}^{n}$ into two path-connected components. Let the point $0 \in \mathbb{R}^{n}$ belong to all bounded components. For arbitrary tetrahedra $t_{1}, \ldots, t_{n-3} \in T$, there exists a 3-plane that passes through 0 and, for $1 \leq i \leq n-3$, contains similarity images of $t_{i}$ with 
common center at 0 such that the vertices of the ith tetrahedron and also the vertices of some tetrahedron negatively homothetic to it and having the same center belong to $K_{i}$.

Proof. It is sufficient to consider the case where $K_{i}$ is an $(n-1)$-dimensional submanifold of $\mathbb{R}^{n}$.

Indeed, for every $\varepsilon>0$ and $1 \leq i \leq n-3$, we construct a subset $K_{i}^{\prime}$ of the $\varepsilon$ neighborhood of $K_{i}$ that contains $K_{i}$ and is a smooth compact $n$-dimensional manifold with boundary $\partial K_{i}^{\prime}$. It is obvious that, for small $\varepsilon>0$, the point 0 lies inside one of the components of the boundary $\partial K_{i}^{\prime}$. If the required tetrahedra exist for a component of the boundary $\partial K_{i}^{\prime}$, then, passing to the limit as $\varepsilon \rightarrow 0$, we construct the required tetrahedra for $K_{i}$.

We prove that, for a typical set of compact connected smooth manifolds $K_{i}$, the number of the required sets of tetrahedra is odd.

In $\left(\mathbb{R}^{n} \backslash 0\right)$, the manifold $K_{i}$ is smoothly isotopic to a manifold that differs from a smoothly embedded sphere $S_{i}^{n-1} \subset\left(\mathbb{R}^{n} \backslash 0\right)$ only in a small neighborhood of its point $x_{i}$ to which we contract all handles of $K_{i}$. If $x_{i}$ is the point of $S_{i}^{n-1}$ farthest from 0 , then the required tetrahedra cannot have vertices close to $x_{i}$ and are inscribed in the corresponding sphere $S_{i}^{n-1}$. As proved above, the number of such configurations is odd. Theorem 8 is proved.

Remark. It is obvious that, in Theorem 8, we can take twisted prisms with given ratio of the base edge to the height instead of pairs of negatively homothetic tetrahedra.

\section{§3. On CONTINuOus Functions on a Euclidean SPHeRE}

Let $M$ be the manifold of positively oriented unit cubes in $\mathbb{R}^{n}$ with center at $0 \in \mathbb{R}^{n}$ and vertices $A_{1}, \ldots, A_{2^{n}}$. The group $G$ of orientation preserving symmetries of a cube acts on $M$ and on the space $\mathbb{R}^{2^{n}}$ by permuting the coordinates of points in the same way as the vertices $A_{1}, \ldots, A_{2^{n}}$ of cubes. Let $L_{1} \subset \mathbb{R}^{2^{k}}$ be the subspace consisting of the points $x \in \mathbb{R}^{2^{k}}$ for which the sum of the coordinates with indices equal to the indices of the vertices of the faces of the highest dimension $n-1$ is constant. Let $L_{2} \subset L_{1}$ be the subspace consisting of the points $x \in \mathbb{R}^{2^{k}}$ for which the sum of the coordinates with indices equal to the indices of the vertices of the faces of dimension $n-2$ is constant. It is easy to prove that $\operatorname{dim} L_{1}-\operatorname{dim} L_{2}=\frac{n(n-1)}{2}$.

Lemma 2. Let $f: M \rightarrow \mathbb{R}^{2^{n}}$ be a continuous mapping that preserves the action of $G$ described above, and let $f(M) \subset L_{1}$. Then $f(M) \cap L_{2} \neq \emptyset$.

Proof. It is sufficient to consider the case where the mapping $f$ is smooth, which is assumed in the sequel. In the remaining cases, the lemma is proved by passing to the limit.

Since $\operatorname{dim} M=\frac{n(n-1)}{2}$, we obtain that, in the general position, the set $f^{-1}\left(L_{2}\right)$ is finite and invariant under the action of $G$. We prove that, in general position, the number $\ell$ of orbits of $G$ in $f^{-1}\left(L_{2}\right)$ is odd.

By standard topological considerations, the residue of $\ell$ modulo 2 does not depend on the choice of a typical mapping $f$ since all such mappings can smoothly be deformed to each other with preserving the action of $G$ (for example, linearly homotopic).

Now, we prove that $\ell \equiv 1 \bmod 2$. Let $m \in M$, and let $f(m)$ be the point whose $i$ th coordinate is the volume of the body that contains the vertex $A_{i}$ of $m$ and is obtained by dividing the body bounded by the heteraxial ellipsoid $E$ centered at $0 \in \mathbb{R}^{n}$ by $n$ pairwise perpendicular hyperplanes passing through 0 and parallel to faces of $m$. We prove that $\ell=1$. 
Let $P_{1}, \ldots, P_{n}$ be pairwise perpendicular hyperplanes passing through the center $0 \in \mathbb{R}^{n}$ of $E$ such that any two of them divide the volume of the region bounded by $E$ into four equal parts. Let $A$ be an affine mapping that transforms the ellipsoid into a sphere. Then the hyperplanes $A\left(P_{1}\right), \ldots, A\left(P_{n}\right)$ are pairwise perpendicular since any two of them divide the volume of the ball into four equal parts, and, therefore, the hyperplanes $P_{1}, \ldots, P_{n}$ are perpendicular to the axes of the ellipsoid.

The fact that the situation in question is general follows from the fact that when the hyperplanes move with nonzero speed, one of the conditions for equality of volumes begins to break with nonzero speed. An equivalent statement is proved in detail in [20]. Lemma 2 is proved.

Now, let $M$ be the manifold of unit $n$-cubes with vertices $A_{1}, \ldots, A_{2^{n}}$ and center at $0 \in \mathbb{R}^{n+1}$, and let $G$ be the group of orientation preserving motions of $\mathbb{R}^{n+1}$ that map a fixed cube to itself.

Lemma 3. Let $f: M \rightarrow \mathbb{R}^{2^{n}}$ be a continuous mapping that preserves the action of $G$. Then $f(M) \cap L_{2} \neq \varnothing$.

Proof. As in Lemma 2, we prove that, for a typical smooth mapping $f$, there is an odd number of positions for the required cube.

The model mapping $f: M \rightarrow \mathbb{R}^{2^{n}}$ is constructed similarly by cutting a heteraxial ellipsoid in $\mathbb{R}^{n+1}$ by $n$ pairwise perpendicular hyperplanes passing through a point distinct from the center of the ellipsoid and lying on one of its axes.

We say that a function $f$ defined on the standard sphere $S^{n-1} \subset \mathbb{R}^{n}$ centered at $0 \in \mathbb{R}^{n}$ is even if, for every point $x \in S^{n-1}$, we have $f(-x)=f(x)$.

Theorem 9. 1. For every continuous function $f$ defined on the standard sphere $S^{n} \subset$ $\mathbb{R}^{n+1}$, there exists an $n$-dimensional cube inscribed in a great $(n-1)$-dimensional sphere in $S^{n}$ such that the sums of the values of $f$ at the vertices of the $(n-2)$-dimensional faces of the cube are the same. 2. For every even continuous function $f$ on the standard sphere $S^{n-1} \subset \mathbb{R}^{n}$, there exists an inscribed $n$-dimensional cube such that all sums of the values of $f$ at the vertices of the $(n-3)$-dimensional faces are equal.

Proof. 1. It is sufficient to apply Lemma 3 to the mapping $F: M \rightarrow \mathbb{R}^{2^{n}}$, where $F\left(A_{1}, \ldots, A_{2^{n}}\right)=\left(f\left(A_{1}\right), \ldots, f\left(A_{2^{n}}\right)\right)$ and $M$ is the manifold of $n$-dimensional cubes with vertices $A_{1}, \ldots, A_{2^{n}}$ inscribed in the great $(n-1)$-dimensional spheres. 2 . It is sufficient to apply Lemma 2 to the manifold $M$ of $n$-dimensional cubes with vertices $A_{1}$, $\ldots, A_{2^{n}}$ inscribed in $S^{n-1}$ and to the mapping of $M$ given by the formula

$$
\left.F\left(A_{1}, \ldots, A_{2^{n}}\right)=\left(f A_{1}\right), \ldots, f\left(A_{2^{n}}\right)\right) .
$$

If the function $f$ is even, then the fact that the sums of the values of $f$ at the vertices of the $(n-2)$-dimensional faces are equal implies that the sums of the values of $f$ at the vertices of the $(n-3)$-dimensional faces are also equal.

Remark. 1. For $n=2$, the first part of the theorem is the Dyson theorem [12] stating that a continuous function on $S^{2} \subset \mathbb{R}^{3}$ takes equal values at the vertices of some square inscribed in a great circle of the sphere.

2. For $n=2$, the second part of the theorem is the theorem from [18] stating that every even function on a two-dimensional sphere takes equal values at the vertices of some cube inscribed in the sphere.

3. For $n=3$, the first part of the theorem states that, for a continuous function $f$ on the sphere $S^{3} \subset \mathbb{R}^{4}$, there exists a three-dimensional cube inscribed in a great two-dimensional sphere of $S^{3}$ such that, for a chess coloring of the vertices of the cube, 
the function $f$ takes equal values at all "white" vertices and equal values at all "black" vertices.

4. For $n=4$, the second part of the theorem states that, for every even continuous function $f$ on the sphere $S^{3} \subset \mathbb{R}^{4}$, there exists a four-dimensional cube inscribed in the sphere such that, for a chess coloring of the vertices of the cube, the function $f$ takes equal values at all "white" vertices and equal values at all "black" vertices.

5. From the proof of Theorem 9, it follows that the first part of the theorem can be generalized to continuous functions on $S_{n}\left(\mathbb{R}^{n+1}\right)$.

Proposition. For every continuous function on the space $S_{n}\left(\mathbb{R}^{n+1}\right)$ of the bundle $S_{n}^{n+1}$ : $S_{n}\left(\mathbb{R}^{n+1}\right) \rightarrow G_{n}\left(\mathbb{R}^{n+1}\right)$, there exists a fiber $S^{n-1} \hookrightarrow S_{n}\left(\mathbb{R}^{n+1}\right)$ in which one can inscribe an $n$-dimensional cube such that the sums of the values of $f$ at the vertices of its $(n-2)$ dimensional faces are equal.

By analogy with Theorems 2 and 7, the following statement is proved.

Theorem 10. Let $n-k$ continuous functions $f_{1}, \ldots, f_{n-k}$ be defined on the total space of the spherization of the tautological bundle $S_{k}^{n}: S_{k}\left(\mathbb{R}^{n}\right) \rightarrow G_{k}\left(\mathbb{R}^{n}\right)$. Then there exists a fiber $S^{k-1} \subset S_{k}\left(\mathbb{R}^{n}\right)$ in which, for $1 \leq i \leq n-k$, one can inscribe a $k$-dimensional cube $Q^{i}$ such that all sums of the values of $f_{i}$ at the vertices of the $(k-2)$-dimensional faces of $Q^{i}$ are equal.

\section{§4. UNIVERSALLY INSCRIBED AND CIRCUMSCRIBED POLYHEDRA IN A EUCLIDEAN SPACE}

Let $A_{1}, \ldots, A_{2^{n}}$ be a cube in $\mathbb{R}^{n}$ centered at 0 . We choose points $B_{1}, \ldots, B_{2^{n}}$ on the rays $0 A_{1}, \ldots, 0 A_{2^{n}}$, respectively. We say that the $2^{n}$-vertex polyhedron obtained has type $Q$. We say that a $2^{n}$-vertex polyhedron of type $Q$ has type $Q_{1}$ if all sums of the lengths of the segments $\left|O B_{i}\right|$ for which the corresponding rays pass through the vertices of the $(n-1)$-dimensional faces of the cube are equal. It is obvious that a polyhedron is of type $Q_{1}$ if and only if $\sum_{i=1}^{2^{n}} \overline{O B_{i}}=\bar{O}$. We say that a $2^{n}$-vertex polyhedron of type $Q$ has type $Q_{i}, 1 \leq i \leq n$, if all sums of the lengths of the segments $\left|O B_{i}\right|$ for which the corresponding rays pass through the vertices of the $(n-i)$-dimensional faces of the cube are equal. It is obvious that $Q \supset Q_{1} \supset Q_{2} \supset \cdots \supset Q_{n}$ and $Q_{n}$ is an $n$-dimensional cube.

Theorem 11. 1. In every smooth convex body $K \subset \mathbb{R}^{n}$, one can inscribe a $2^{n}$-vertex polyhedron of type $Q_{2}$.

2. In every centrally symmetric convex body, one can inscribe a $2^{n}$-vertex polyhedron of type $Q_{3}$ with the same center of symmetry.

Proof. As above, we prove that, for a smooth convex body $K$ in general position, the number of the required $2^{n}$-vertex polyhedra is odd.

For a smooth convex body $K \subset \mathbb{R}^{n}$, we denote by $M(K)$ the space of $2^{n}$-vertex polyhedra of type $Q_{1}$ inscribed in $K$. It is clear that, for a typical smooth convex body, $M(K)$ is a smooth compact $n(n-1) / 2$-dimensional submanifold of the manifold of all positions of the cube $A_{1}, \ldots, A_{2^{n}}$. It is easy to prove that if $K$ is a ball or if $K$ is a smooth body in $\mathbb{R}^{n}$ that is $C^{1}$-close to a ball, then the manifold $M(K)$ is diffeomorphic to the group $S O(n)$ of the orientation preserving rotations of $\mathbb{R}^{n}$ about a fixed point since the centers of the required polyhedra necessarily coincide with the center of the ball $K$.

We consider the mapping $F: M(K) \rightarrow \mathbb{R}^{2^{n}}$ given by the formula $F\left(B_{1} \cdots B_{2^{n}}\right)=$ $\left(\left|O B_{1}\right|, \ldots,\left|O B_{2^{n}}\right|\right)$. If $K$ is sufficiently $C^{1}$-close to a ball, then $M(K) \cong S O(n)$. By Lemma 2 , an odd number of $2^{n}$-vertex polyhedra of $Q_{2}$ is inscribed in $K$ in general position. Since two arbitrary smooth bodies in $\mathbb{R}^{n}$ can be smoothly deformed to each 
other in the class of such bodies, by standard considerations we obtain that, in general position, the number of required inscribed $2^{n}$-vertex polyhedra of type $Q_{2}$ is odd since, by smoothness, the center of the $2^{n}$-vertex polyhedron in question cannot approach the boundary of the body during the smooth deformation.

Passing to the limit, we obtain that, in every smooth convex body, one can inscribe a $2^{n}$-vertex polyhedron of type $Q_{2}$ since, by the above reasons, the center of an inscribed $2^{n}$-vertex polyhedron cannot approach the boundary of the body.

From the proof of the first part of the theorem, we see that every centrally symmetric convex body in $\mathbb{R}^{n}$ is circumscribed about a $2^{n}$-vertex polyhedron of type $Q_{2}$ with the same center. By central symmetry, this polyhedron automatically has type $Q_{3}$.

Remarks. 1. For $n=2$, the theorem says that, in a smooth convex figure in a plane, one can inscribe a square. Shnirel'man [11] proved this statement for smooth plane Jordan curves.

2. For $n=3$, the theorem says that, in a smooth convex body in $\mathbb{R}^{3}$, one can inscribe a regular equiaugmented tetrahedron (Section 2). In Section 2 of the present paper, we proved a more general statement, from which it follows that, in every smoothly embedded sphere $S^{2} \hookrightarrow \mathbb{R}^{3}$, an eight-vertex polyhedron of type $Q_{2}$ can be inscribed.

3. Up to similarity, the $2^{n}$-vertex polyhedra of type $Q_{2}$ form a $\left(2^{n}-\frac{n(n+1)}{2}-1\right)$-parametric family of $2^{n}$-vertex polyhedra.

Similarly to Theorem 8 , the following result is proved.

Theorem 12. Let $K_{1}, \ldots, K_{\ell}$ be compact subsets in $\mathbb{R}^{n}$ each of which divides $\mathbb{R}^{n}$ into two path-connected components so that the point $0 \in \mathbb{R}^{n}$ belongs to all bounded components and $\ell<n$. Then there is an $(n-\ell)$-dimensional plane passing through the point 0 in which, for each $1 \leq i \leq \ell$, lies an $(n-\ell)$-dimensional polyhedron of type $Q_{2}$ with center at 0 and the vertices belonging to the set $K_{i}$.

We consider the dual problem concerning circumscribed polyhedra.

Let $1 \leq i \leq n$. By a polyhedron of type $Q_{i}^{*}$, we mean the intersection of $2^{n}$ half-spaces of $\mathbb{R}^{n}$ containing the point $0 \in \mathbb{R}^{n}$ and bounded by the hyperplanes passing through the vertices $B_{j}$ of a $2^{n}$-vertex polyhedron of type $Q_{i}$ and perpendicular to the lines $O B_{j}$ (where 0 is the center of the polyhedron of type $Q_{i}$ in question).

Similarly to Theorem 11, the following result is proved.

Theorem 13. 1. About every bounded subset of $\mathbb{R}^{n}$ a polyhedron of type $Q_{2}^{*}$ can be circumscribed.

2. About every centrally symmetric bounded subset of $\mathbb{R}^{n}$ a polyhedron of type $Q_{3}^{*}$ with the same center of symmetry can be circumscribed.

We note that, in dimension 4, the second part of Theorem 11 says that, in every centrally symmetric convex body in $\mathbb{R}^{4}$, one can inscribe a 16 -vertex polyhedron obtained from a four-dimensional cube with a chess coloring by moving eight "black" vertices to the center of the cube by the same distance. The second part of Theorem 13 states that, about every bounded centrally symmetric subset in $\mathbb{R}^{4}$, one can circumscribe a 16 -vertex polyhedron obtained from a regular octahedron (a polyhedron dual to a cube) with a chess coloring by the parallel translation of the planes of all "black" faces toward the center of the polyhedron by the same distance.

In conclusion, we consider polyhedra circumscribed about a convex subset of $\mathbb{R}^{n}$ of constant width, i.e., with a constant distance between the pairs of the hyperplanes of support.

Theorem 14. About every convex body $K \subset \mathbb{R}^{n}$ of constant width, one can circumscribe $a\left(2^{n}+2 n\right)$-vertex polyhedron bounded by the faces of a cube $Q$ and by $2^{n-1}$ pairs of 
hyperplanes perpendicular to the diagonals of the cube such that all sums of distances from the center of the cube to the midpoint of the segment cut out from a diagonal by the pair of hyperplanes perpendicular to it counted along the diagonals going to the vertices of each $(n-3)$-dimensional face of the cube $Q$ are equal.

Proof. It is sufficient to prove that about every convex body $K \subset \mathbb{R}^{4}$ of constant width one can circumscribe a cube centered at 0 such that all sums of distances from 0 to the midpoints of the orthogonal projections of $K$ onto the diagonals of the cube counted for the diagonals going to the vertices of an arbitrary $(n-3)$-dimensional face of the cube are equal.

To each positively oriented cube $A_{1} \cdots A_{2^{n}}$ circumscribed about a convex body $K \subset$ $\mathbb{R}^{n}$ of constant width, we assign the point in $\mathbb{R}^{2^{n}}$ whose $i$ th coordinate is equal to the distance from the center 0 of the cube to the midpoint of the orthogonal projection of $K$ to the line $O A_{i}$. Applying Lemma 2 to the above mapping of the manifold of circumscribed cubes to $\mathbb{R}^{2^{n}}$ and taking into account the fact that the coordinates of the points corresponding to the vertices of the cube symmetric with respect to the center 0 are equal, we obtain the required statement.

Remark. For $n=2$ and $n=3$, Theorem 14 does not give any new information.

For $n=4$, up to similarity, the 24-hedra circumscribed about a convex body $K \subset \mathbb{R}^{4}$ of constant width that are obtained by Theorem 14 form a two-parameter family. The two parameters are the distances of the midpoint of the orthogonal projection of $K$ to a diagonal of the cube circumscribed about $K$ from the center 0 of the cube.

\section{REFERENCES}

[1] B. Knaster, Problem P 4, Colloq. Math. 1 (1948), no. 1, 30-31.

[2] E. E. Floyd, Real-valued mappings of spheres, Proc. Amer. Math. Soc. 6 (1955), 957-959. MR0073978 (17:518b)

[3] V. V. Makeev, Some properties of continuous mappings of spheres and problems in combinatorial geometry, Geometric Questions in the Theory of Functions and Sets, Kalinin. Gos. Univ., Kalinin, 1986, pp. 75-85. (Russian) MR1027885

[4] W. Chen, Counterexamples to Knaster's conjecture, Topology 37 (1998), 401-405. MR1489211 (99c:55002)

[5] B. S. Kashin and S. J. Szarek, The Knaster problem and the geometry of high-dimensional cubes, C. R. Math. Acad. Sci. Paris 336 (2003), 931-936. MR1994597 (2005c:46017)

[6] A. Hinrichs and C. Richter, New counterexamples to Knaster's conjecture, Preprint, 2003.

[7] V. V. Makeev, Affine-inscribed and affine-circumscribed polygons and polyhedra, Zap. Nauchn. Sem. S.-Peterburg. Otdel. Mat. Inst. Steklov. (POMI) 231 (1995), 286-298; English transl., J. Math. Sci. (New York) 91 (1998), no. 6, 3518-3525. MR1434300 (98b:52004)

[8] - Three-dimensional polytopes inscribed in and circumscribed about compact convex sets, Algebra i Analiz 12 (2000), no. 4, 1-15; English transl., St. Petersburg Math. J. 12 (2001), no. 4, 507-518. MR1793615 (2001k:52017)

[9] _ Three-dimensional polytopes inscribed in and circumscribed about compact convex sets. II, Algebra i Analiz 13 (2001), no. 5, 110-133; English transl., St. Petersburg Math. J. 13 (2002), no. 5, 791-807. MR1882865 (2003g:52017)

[10] _ On quadrangles inscribed in a closed curve, Mat. Zametki 57 (1995), no. 1, 129-132; English transl., Math. Notes 57 (1995), no. 1-2, 91-93. MR1339220 (96d:51029)

[11] L. G. Shnirel'man, On certain geometrical properties of closed curves, Uspekhi Mat. Nauk Vyp. 10 (1944), 34-44. (Russian) MR0012531 (7:35c)

[12] F. J. Dyson, Continuous functions defined on spheres, Ann. of Math. (2) 54 (1951), 534-536. MR0044620 (13:450f)

[13] E. Fadell and S. Husseini, An ideal-valued cohomological index theory with applications to BorsukUlam and Bourgin-Yang theorems, Ergodic Theory Dynam. Systems 8 (1988), Charles Conley Memorial Issue, 73-85. MR0967630 (89k:55002)

[14] R. T. Živaljević and S. T. Vrećica, An extension of the ham sandwich theorem, Bull. London Math. Soc. 22 (1990), 183-186. MR1045292 (91j:52006) 
[15] V. Dol'nikov, Transversals of families of sets in $\mathbb{R}^{n}$ and a relationship between Helly and Borsuk theorems, Mat. Sb. 184 (1993), no. 5, 111-132; English transl., Russian Acad. Sci. Sb. Math. 79 (1994), no. 1, 93-107. MR1239754 (94g:52005)

[16] _ A generalization of the sandwich theorem, Mat. Zametki 52 (1992), no. 2, 27-37; English transl., Math. Notes 52 (1992), no. 1-2, 771-779 (1993). MR1187871 (93j:28002)

[17] H. Griffiths, The topology of square pegs in round holes, Proc. London Math. Soc. (3) 62 (1991), 647-672. MR1095236 (92h:55004)

[18] T. Hausel, E. Makai, and A. Szücz, Inscribing cubes and covering by rhombic dodecahedra via equivariant topology, Mathematika 47 (2000), 371-397 (2002). MR1924512 (2003f:52010)

[19] S. A. Kakutani, A proof that there exists a circumscribing cube around any bounded closed convex set in $R^{3}$, Ann. of Math. (2) 43 (1942), 739-741. MR0007267 (4:111i)

[20] B. A. Rattray, An antipodal-point, orthogonal-point theorem, Ann. of Math. (2) 60 (1954), 502-512. MR0065153 (16:388g)

Department of Mathematics and Mechanics, St. Petersburg State University, UniverSitetski ̌ Prospect 28, Stary ̌ Peterhof, St. Petersburg 198904, Russia

E-mail address: mvv57@inbox.ru

Received 20/MAY/2005

Translated by B. M. BEKKER 